\title{
Manifestações orais e condutas em pacientes oncológicos pediátricos: revisão da literatura
}

\author{
Oral manifestations and conduct in pediatric \\ oncologic patients: literature review
}

\author{
Fabrício Campos Machado* \\ Marília Rodrigues Moreira** \\ Mirna Scalon Cordeiro*** \\ Thiago de Amorim Carvalho****
}

\begin{abstract}
RESUMO
Objetivo: O presente trabalho trata-se de uma revisão da literatura, que aborda as principais manifestações orais, e possibilidades terapêuticas no âmbito odontológico, nos pacientes oncológicos pediátricos.

Material e métodos: Neste contexto foram revisados 20 artigos nacionais e internacionais, levantados a partir de bases de dados PUBMED, LILACS, BVS, SCIELO, BBO, com utilização das palavras chave: câncer, oncologia, oncologia pediátrica e a associação odontologia e oncologia pediátrica.

Resultados: o câncer é a segunda causa de morte entre crianças e adolescentes, e as manifestações orais estão presentes tanto por decorrência da doença, quanto pelo tratamento utilizado, sendo os mais comuns a quimioterapia e a radioterapia. A mucosite é a manifestação oral mais frequente em crianças submetidas à terapia antineoplásica e leva a prejuízos importantes ao prognóstico e à qualidade de vida dos pacientes. Outras alterações importantes descritas são a osteorradionecrose, a cárie de radiação e a doença periodontal. Terapias conservadoras clássicas como a adequação químico-mecânica do meio bucal previamente ao tratamento quimio/radioterápico, bem como terapias modernas como a laserterapia de baixa intensidade são possíveis para melhoria do quadro das principais alterações.

Conclusão: $O$ diagnóstico precoce e o manejo terapêutico das manifestações bucais associadas aos pacientes oncológicos pediátricos é essencial para a manutenção da qualidade de vida e consolidação de uma boa saúde bucal em crianças submetidas a tratamento antineoplásico. A adequada assistência odontológica exige uma conduta integrada, conhecimento técnico científico de técnicas modernas (como o uso de laser terapêutico) e visão holística do paciente.
\end{abstract}

Palavras chave: Odontologia, Oncologia, Odontopediatria.

\begin{abstract}
Objective: The presente study is a literature review, which approach the main oral manifestations, and therapeutic possibilities in dentistry domains, on oncologic pediatric patients

Material and Methods: In this context, 20 articles was reviwed from database of PUBMED, LILACS, BVS, Scielo and BBO, using the keywords cancer, oncology, pediatric oncology, and the association between dentistry and pediatric oncology

Results: Cancer is the second death cause among children and teenagers, and oral manifestations are present because the illness, and its treatment with chemotherapy and radiotherapy. Mucositis would be the oral manifestation more frequent in children undergoing antineoplastic therapy with important damages to the prognosis and the patient's quality of life. Other important changes would be osteoradionecrosis, radiation caries and periodontal disease. Classical conservative therapies such as the chemical-mechanical
\end{abstract}

* Acadêmico de Graduação em Odontologia do Centro Universitário do Triângulo - Uberlândia- MG

** Professora do Curso de Graduação em Odontologia do Centro Universitário de Patos de Minas - Patos de Minas-MG

*** Professor do Curso de Graduação em Odontologia do Centro Universitário do Triângulo - Uberlândia-MG

Autor para correspondência: Thiago de Amorim Carvalho - dr.thiagocarvalhocd@gmail.com 
suitability of the buccal environment prior to chemo / radiotherapeutic treatment as well as modern therapies such as low intensity laser therapy are possible to improve the picture of the main alterations.

Conclusion: Early diagnosis and therapeutic management of oral manifestations associated with pediatric oncology patients is essential for the maintenance of quality of life and the consolidation of good oral health in children undergoing antineoplastic treatment. Appropriate dental care requires integrated management, scientific technical knowledge of modern techniques (such as the use of therapeutic laser), and holistic view of the patient.

Keywords: Dentistry, Oncology, Children dentistry.

\section{INTRODUÇÃo}

Com a inversão epidemiológica, proporcionada principalmente pelo advento dos programas de vacinação e do uso e desenvolvimento dos antibióticos, as doenças crônicas não transmissíveis passam a figurar como as mais frequentes na população. As crianças não ficaram alheias a esta inversão, sendo que as doenças agudas frequentes neste grupo populacional, como varicela, rubéola, paralisia infantil, entre outras, passam a figurar no hall das doenças erradicadas ou pouco frequentes, e as doenças como cardiopatias e câncer aparecem como causas importantes de morbimortalidade infantil.

As neoplasias malignas têm sido consideradas um problema de saúde pública ${ }^{1}$, e é consenso que tais neoplasias em crianças e adolescentes devem ser abordadas de forma diferente de suas manifestações em adultos, dada a diferença entre os locais primários, a origem histológica e comportamento clínico. $\mathrm{O}$ câncer em crianças e adolescentes apresenta crescimento mais rápido, maior capacidade de invasão, porém e de forma até tranquilizadora, tende a responder melhor ao tratamento quimioterápico ${ }^{2,3}$.

No curso clínico de uma doença grave como o câncer, todos os sistemas tendem a ser afetados, inclusive o sistema estomatognático. Por ter ampla capacidade de renovação celular, a mucosa oral é um dos sítios mais acometidos pela ação dos fármacos quimioterápicos, posto que, estes têm entre seus efeitos a inibição da multiplicação celular, que é extremamente presente nas neoplasias malignas. Os dentes podem ser afetados pela xerostomia causada pela medicação (que pode acarretar maior incidência de cáries de progressão rápida), e pela radioterapia quando esse procedimento é direcionado a região da cabeça e do pescoço. O tecido periodontal pode também ser acometido e apresentar recessões e sangramento. Na mucosa é frequente o aparecimento de numerosas úlceras, extensas, de longa duração e que apresentam sintomatologia dolorosa aguda, comumente chamadas mucosites ${ }^{4}$.

O cirurgião dentista deve ser parte da equipe multiprofissional pois é o profissional melhor preparado técnica e cientificamente para lidar com as alterações orais relacionadas ao câncer ${ }^{5}$. As manifestações orais decorrentes do tratamento antineoplásico, bem como o uso de tecnologia moderna como o laser terapêutico de baixa frequência e outros meios conservadores na abordagem das mucosites, além da capacidade de motivação e elaboração de programas preventivos e educativos, serão os temas desenvolvidos nesta revisão de literatura.

\section{Material e métodos}

Este trabalho foi desenvolvido por meio de revisão da literatura, com pesquisa em bases de dados nacionais (Scielo, Biblioteca Virtual em Saúde) e internacionais (LILACS/ BIREME e PubMed). Foram utilizadas as palavras chave: oncologia pediátrica, pediatria, oncologia, odontologia, e suas relações bem como seus correspondentes em inglês pediatric oncology, pediatry, oncology, dentristry. Com 
essa abordagem foram selecionados 34 artigos, nos quais 20 se enquadraram na metodologia proposta: revisão de literatura com contexto direcionado para manifestações orais do tratamento oncológico em crianças. Os artigos foram excluídos quando não se enquadravam na delimitação do tempo proposto (últimos quinze anos 2001 - 2016), e quando o tema abordado era muito restrito ao tipo de neoplasia, não considerando a abordagem odontológica. A seleção foi feita por meio da leitura do título, resumo e abstract dos artigos.

Tabela 1 - Estudos e manifestações orais descritas em pacientes com câncer

\begin{tabular}{|c|c|}
\hline Autores (ano) & $\begin{array}{l}\text { Manifestações orais } \\
\text { mais frequentes, } \\
\text { por tratatamento } \\
\text { oncológico radio/ } \\
\text { quimioterápico }\end{array}$ \\
\hline TORRES - PEREIRA (2002)* & Mucosite \\
\hline SEGER et al. (2002)* & Mucosite \\
\hline SCHWARTZ \& KAGAN (2002) & Osteorradionecrose \\
\hline DE CASTRO et al. (2002) & Osteorradionecrose \\
\hline MARTINS et al. (2002) & $\begin{array}{l}\text { Candidose, trismo, } \\
\text { odontalgias }\end{array}$ \\
\hline ELTING et al. (2003)* & Mucosite \\
\hline SONIS et al. (2004)* & Mucosite \\
\hline FONSECA $(2004)^{*}$ & Mucosite \\
\hline PEREIRA et al. (2005) & Mucosite, parestesia \\
\hline GRIMALDI et al. (2005) & Osteorradionecrose \\
\hline FIGLIOLA et al. $(2006)^{*}$ & Mucosite \\
\hline ROSALES (2009) & Osteorradionecrose \\
\hline MEURMAN et al. (2010) & Cárie por radiação \\
\hline CARRILLO $(2010)^{*}$ & Mucosite \\
\hline MILLER et al. (2012)* & Mucosite \\
\hline LINO et al (2013)* & Mucosite \\
\hline MUFEED et al. (2015) & Cárie por radiação \\
\hline MELO JÚNIOR (2015) & Mucosite \\
\hline MONTEIRO et al. (2016)* & $\begin{array}{l}\text { Candidose, trismo, } \\
\text { mucosite }\end{array}$ \\
\hline
\end{tabular}

\begin{tabular}{l|l}
\hline ALBUQUERQUE et al. (2016)* & Mucosite
\end{tabular}

$\left.{ }^{*}\right)$ abordam especificamente o câncer infantil ou tratam deste no corpo do artigo

\section{Resultados e discussão}

Artigos relacionados ao câncer infantil e mais especificamente sobre as manifestações orais, estão presentes na literatura desde o início da década de 90 , referindo sobre a alta taxa de mortalidade desta doença na década de 60 nos Estados Unidos, já que nesta época esta afecção era considerada fatal. Os artigos e suas respectivas abordagens nas manifestações orais estão contidos na tabela 1.

Dada sua gravidade, o câncer se configura como uma das doenças que mais oneram recursos financeiros no contexto da saúde, já que o diagnóstico e tratamento em geral são realizados por meios altamente tecnológicos 5 .

No contexto da pediatria trata-se de uma doença considerada rara, mas ainda assim no Brasil corresponde a segunda principal causa de mortalidade entre crianças e adolescentes de 0 a 19 anos $^{6}$. O diagnóstico do câncer infantil é um desafio para pediatras e outros profissionais da saúde, incluindo o cirurgião dentista, posto a inespecificidade dos sinais e sintomas e o diagnóstico diferencial desta doença, com outras comuns na infância, inclusive com os traumatismos ${ }^{6}$. São comuns sinais como manchas avermelhada e arroxeadas, aumento volumétrico de membros inferiores e superiores, bem como febre e perda de apetite, associado com linfadenopatia ${ }^{7,8}$.

Os tipos de câncer mais frequentemente diagnosticados em crianças e adolescentes são bastante controversos dado a grande quantidade de centros especializados no Brasil e no mundo, porém as leucemias e os linfomas são aqueles que mais ocorrem nos registros da literatura como sendo os tipos de neoplasias malignas mais frequentes neste público ${ }^{9}$. Com o aumento da sobrevida e a diminuição das taxas de mortalidade de pacientes pediátricos, as chances de que estes apresentem alterações 
sistêmicas decorrentes dos efeitos adversos do tratamento, incluindo as manifestações orais, aumentam consideravelmente, e podem acarretar em uma piora no quadro clínico com consequente piora no prognóstico, dado o aumento do risco de infecções e aumento do tempo de internação ${ }^{10,11,12}$.

As alterações bucais estão divididas em alterações primárias, secundárias e terciárias. As alterações primárias são aquelas causadas diretamente pela infiltração dos tecidos orais pelos tumores da região da cabeça e do pescoço, se apresentado clinicamente como nódulos ou placas brancas gengivais com bordas avermelhadas, causadas pela infiltração do tecido ósseo e gengival respectivamente. As alterações secundárias estão relacionadas às doenças próprias do curso clínico das neoplasias, como anemia, trombocitopenia e granulocitopenia, que se apresentam clinicamente como palidez das mucosas, dada a anemia crônica, petéquias hemorrágicas, causadas pela trombocitopenia, úlceras neutropênicas e ainda doença periodontal dada a dimunição dos fatores de resistência do hospedeiro. As alterações terciárias estão diretamente relacionadas ao tratamento antineoplásico e que se manisfestam clinicamente como mucosite, parestesia e infecções oportunistas dada a imunossupressão ${ }^{13,14}$.

Dentreasalteraçõesbucais, as mucosites, talvez por sua gravidade, comprometimento do organismo e complicação do quadro clínico geral, apresentam na literatura uma riqueza de possibilidades terapêuticas. A mucosite trata-se da manifestação oral mais comum em pacientes sob tratamento antineoplásico ${ }^{15}$. A manifestação clínica da mucosite se dá geralmente, entre cinco a sete dias após o início da quimioterapia, seu aparecimento e curso clínico estão relacionados a fatores como idade do paciente (quanto mais jovem maior a chance de se apresentar esta complicação), a higiene oral deficiente e o grau de comprometimento da medula. ${ }^{16}$

A patogenia das mucosites envolve eventos inflamatórios complexos (como angiogênese e a comunicação por meio de citocinas para o conjuntivo subepitelial), e a própria quimioterapia, que reduz a capacidade de proliferação celular na camada basal do epitélio acarretando maior descamação e ausência de reparo. Este evento pode ser explicado em função da angiogênese aumentar a concentração do fármaco quimioterápico naquela região, evoluindo inicialmente para o aparecimento de um eritema, que posteriormente evolui para uma úlcera bastante dolorosa, que quando em presença de grande concentração de biofilme oral pode se tornar infectada e gerar diversas complicações nutricionais (dificuldade de alimentação) e para o bem estar e qualidade de vida do paciente ${ }^{17}$.

O tratamento das mucosites pode ser feito com a ajuda de diversas terapias, porém, devem ser de rápido efeito, dada a espoliação do paciente durante o quadro desta alteração patológica. Em crianças geralmente é feito de forma conservadora, com a remoção de focos infecciosos previamente ao inicio da quimioterapia, bem como o uso de clorexidina $0,12 \%$ como adequação química do meio bucal, e ainda mais recentemente o uso do laser de baixa potência como meio físico de fortalecimento das camadas epiteliais, prevenindo a recidiva e estimulando a proliferação epitelial a partir da camada basal, além da ação moduladora na resposta inflamatória da cavidade oral 15,18,19. Os lasers He-Ne (Hélio-Neônio) e GaAIAs (Gálio-Alumínio- Arsênio) são os tipos que apresentam melhores resultados para mucosites orais ${ }^{19}$.

Outras manifestações orais importantes da quimioterapia são a xerostomia, úlceras, doença de Behçet; disfagia, disgeusia, 
odinofagia; a palidez na mucosa, boca seca, a gengivite ulcerativa necrosante (GUN), a odontalgia, as infecções fúngicas (com aumento da incidência de candidose), as infecções causadas por bactérias e vírus (por exemplo herpes) além de alterações no paladar, o trismo muscular; as doenças periodontais envolvendo principalmente o ligamento periodontal, o sangramento gengival e as hemorragias ${ }^{20}$.

Em relação à radioterapia esta tem sido uma das modalidades de tratamento mais amplamente utilizada no tratamento das neoplasias malignas, isoladamente ou associada à quimioterapia e cirurgia ${ }^{21}$. Assim como toda modalidade terapêutica a radioterapia também acarreta efeitos colaterais, devido a hipocelularidade e a hipóxia tecidual ocasionadas pela radiação e quando feita em região de cabeça e pescoço, as principais manifestações orais incluem a hipossalivação, mucosite, infecção por espécies de Candida, trismo, alterações no paladar, cárie de radiação e a osteorradionecrose ${ }^{22}$.

A cárie por radiação é uma doença altamente destrutiva, sem localização específica e apresenta como complicação a diminuição da sensibilidade dolorosa nas regiões afetadas pela fibrose e atrofia dos tecidos pulpares ${ }^{23}$. Um dos principais fatores colaboradores para o desenvolvimento desta alteração seria a hipossalivação, desfavorecendo a remineralização, a auto limpeza bucal e ainda o controle do $\mathrm{pH}$ do meio bucal ${ }^{24}$.

A osteorradionecrose é uma complicação grave da radioterapia em região da cabeça e do pescoço ${ }^{25}$. É ocasionada pela redução do potencial de vascularização dos tecidos. As consequentes condições hipovascular e hipóxica colocam em risco a atividade celular, formação de colágeno e capacidade curativa de ferida. Com os vasos alterados o fluxo sangüíneo diminui, bem como os nutrientes e as células de defesa. Sem nutrientes e sem defesa toda a estrutura dos ossos maxilar e mandibular sofre degeneração ${ }^{26,27}$. Desta forma toda e qualquer intervenção odontológica deve ser muito bem planejada no pós radioterapia, já que o tratamento da osteorradionecrose é complexo e oneroso, e o prognóstico não é favorável ${ }^{25}$.

Apresentadas as manifestações orais mais frequentes decorrentes tanto da própria patogenia do câncer, bem como as alterações causadas pela terapia antineoplásica, a participação do cirurgião dentista na equipe multiprofissional se torna fundamental e indispensável.

Dado a maior incidência de problemas bucais pós tratamento oncológico, uma avaliação prévia ao tratamento quimioterápico e radioterápico é extremanente necessária com remoção dos focos infecciosos, adequação de meio bucal abrangente, com remoção das lesões cariosas e aplicação de ionômero de vidro, exodontia de dentes decíduos em situação de risco, ou próximos de sofrerem rizólise, uso de clorexidina 0,12 \% como adequação química do meio bucal e aplicação de flúor no intuito de fortalecer as estruturas mineralizadas ${ }^{28,29}$. Além disso estudos $^{30}$ demonstram que a incidência de osteorradionecrose em pacientes que receberam atendimento odontológico prévio ao tratamento oncológico, diminui.

Ainda em relação ao tratamento, cabe salientar que a anamnese é fundamental e determinante para o planejamento e condução segura da assistência odontológica. Informações sobre a data do término do tratamento e a solicitação de exames complementares é geralmente necessária, principalmente um hemograma, um coagulograma e um teste de glicemia em jejum, de acordo com os procedimentos que serão executados. Além disso a solicitação de pareceres médicos se torna indispensável ${ }^{30}$. 
Programas de prevenção e promoção em saúde bucal permanentes são ferramentas importantes para a profilaxia de complicações orais decorrentes da terapia antineoplásica, já que durante o curso da doença, tanto a criança, quanto os responsáveis estão sensibilizados e abertos a receberem informações, que possam de certa forma colaborar durante o processo complexo de tratamento oncológico. A participação do cirurgião dentista na equipe multiprofissional em centros oncológicos (neste contexto o odontopediatra) seria uma forma de abrandar as alterações bucais decorrentes do câncer e seu tratamento, podendo melhorar o prognóstico e a qualidade de vida do paciente ${ }^{28}$.

A literatura é clara acerca das relações entre manifestações orais, neoplasias malignas e terapia antineoplásica. A partir da revisão da literatura pode-se corroborar a necessidade do cirurgião dentista em todas as etapas do tratamento e como integrante permanente da equipe multiprofissional de cuidados ao paciente oncológico pediátrico.

A mucosite conforme todos os trabalhos citados ${ }^{12,14,21}$ é a manifestação oral mais comum, que causa os maiores prejuízos durante $\mathrm{O}$ tratamento antineoplásicos. $\mathrm{E}$ acomete cerca de $80 \%$ das crianças sob quimioterapia ${ }^{17}$. Uma das modalidades mais aceitas e modernas para sua prevenção e tratamento seria o uso do laser de baixa intensidade, com comprimento de onda acima de $900 \mathrm{~nm}$, infravermelho, com aplicações diárias um dia antes do início da quimioterapia e durante todo o curso do ciclo quimioterápico/ radioterápico $15,17,18,19$.

Sobre a osteorradionecrose é consenso que se trata de uma alteração grave decorrente do tratamento antineoplásico e que cuidados para o tratamento odontológico devem ser tomados mesmo depois do término da radioterapia, dada a controversa na literatura sobre o prazo mínimo para a realização de intervenções odontológicas, com citações de prazo de aproximadamente cinco anos, embora a necessidade do parecer médico mesmo após este prazo ainda seja indispensável 25,26,27,30.

A necessidade de programas permanentes de promoção e prevenção em saúde bucal são fundamentais para os pacientes que serão submetidos à terapia antineoplásica para garantir mais qualidade de vida e maior segurança nas intervenções caso essas sejam necessárias.

O conhecimento do dentista sobre solicitação e interpretação de exames também se faz presente na literatura pesquisada, sendo o resultado dos exames de coagulação e hemograma determinantes na decisão da profilaxia antibiótica e até mesmo no melhor momento de intervenções eletivas. Neutrófilos em concentração menor que 1000 células $/ \mathrm{mm}^{3}$ contraindicam o tratamento eletivo, bem como em níveis de 1000 a 2000 células $/ \mathrm{mm}^{3}$ exigem a solicitação de parecer médico e profilaxia antibiótica. Se o nível plaquetário estiver entre 40.000 e 75.000 células $/ \mathrm{mm}^{3}$, pode ser necessário uma transfusão de plaquetas previamente ao procedimento. Estes dados ainda mostram a necessidade uma equipe multiprofissional e com boa comunicação para o atendimento de excelência ao paciente oncológico pediátrico ${ }^{25,30}$.

\section{Conclusão}

Várias são as manifestações bucais que podem estar presentes em pacientes oncológicos pediátricos, sendo a mucosite a mais prevalente. Para tratar adequadamente cada alteração, e evitar o aparecimento de complicações, o odontólogo precisa estar alinhado com o conhecimento científico atual, estar inserido em equipes multidisciplinares e ter uma visão holística e integralizada sobre o paciente. A implementação de programas preventivos individualizados também é essencial para a manutenção da qualidade de 
vida e bem-estar desses pacientes, durante e após a terapia anti-neoplásica.

\section{REFERÊNCIAS}

1. Brasil. Ministério da Saúde. Instituto Nacional do Câncer. Câncer na criança e no adolescente no Brasil: dados do registro de base populacional e de mortalidade. Rio de Janeiro, 2008.

2. Brasil. Ministério da Saúde. Instituto Nacional de Câncer. Câncer no Brasil: dados dos registros de base populacional. Rio de Janeiro, 2003.

3. Brasil. Instituto Nacional do Câncer. Estimativa 2014: Incidência de Câncer no Brasil / Instituto Nacional de Câncer José Alencar Gomes da Silva, Coordenação de Prevenção e Vigilância, 2014.

4. Albuquerque, RA, Morais VLL, Sobral APV. Odontologic protocol of attendance the pediatric oncology patients: review of literature. Rev Odontol UNESP. 2007, 36(3):275-280

5. Osterne, RLV. et al. Saúde bucal em portadores de neoplasias malignas: estudo clínicoepidemiológico e análise das necessidades odontológicas de 421 pacientes. Revista Brasileira de Cancerologia 2008. 54(3): 22126.

6. Ribeiro, IAL., Valença, AMG., Bonan, P R F. Odontologia na oncologia pediátrica. 2016. João Pessoa, Ideia.

7. Haimi M, Peretz Nahum M, Ben Arush MW. Delay in diagnosis of children with cancer: a retrospective study of 315 children. Pediatr Hematol and Oncol. 2004 21(1): 37-48.

8. Dang-Tan T, Franco EL. Diagnosis delays in childhood cancer: a review. Cancer.2007 110(4):703-13.

9. Brasil. Instituto Nacional do Câncer. Câncer infantil. 2012.

10. Braga PE., Latorre MRD, Curado MP. Câncer na infância: análise comparativa da incidência, mortalidade e sobrevida em Goiânia (Brasil) e outros países. Cad Saúde Pública. 2002 (18)1: 33-44.

11. Carrillo C, Fava M, Vizeu H, Soares-Junior LA, Odone Filho V. Dental approach in the pediatric oncology patient: characteristics of the population treated at the dentistry unit in a
Pediatric Oncology Brazilian Teaching Hospital. Clinics.2010 65(6): 569-73.

12. Sonis ST. The pathobiology of mucositis. Nat Rev Cancer.2005 4: 277- 84.

13. Pereira, J V et al. Avaliação de Streptococcus mutans e velocidade do fluxo salivar em pacientes com câncer de cabeça e pescoço submetidos à quimioterapia e radioterapia. Pesq Bras Odontoped Clin Integr, João Pessoa. 2008. 8(3):295-299.

14. Elting, $L$ et al. The burdens of cancer therapy: clinical and economic outcomes of chemotherapy-induced mucositis. Cancer, New York. 2003. 98(7):1531-1539.

15. Sandoval, $R \mathrm{~L}$ et al. Management of chemio and radiotherapy induced oral mucositis with low energy laser: initial results of A.C. Camargo hospital. J. Appl. Oral. Sci.2003. 11(4): 337-41.

16. Sonis, ST et al. Perspectives on cancer therapy induced mucosal injury. Cancer, New York. 2004. 100(9): 1995-2025.

17. Miller, MM, Donald, DV, Hagemann, TM. Prevention and treatment of oral mucositis in children with cancer. J Pediatr Pharmacol Ther. 2012. 17(4):340-350

18. Lino MDMC et al . Laser phototherapy as a treatment for radiotherapy-induced oral mucositis. Braz Dent J. Ribeirão Preto. 2013. 22(2).

19. Chow, RT, Johnson, MI, Lopes-Martins, RA, Bjordal, JM. Efficacy of low-level laser therapy in the management of neck pain: a systematic review and meta-analysis of randomised placebo or active-treatment controlled trials. Lancet. 2009. 5(374) Suppl. 9705: 1897-908.

20. Martins, ACM, Caçador, NP, Gaeti, WP. Complicações bucais da quimioterapia antineoplásica. Acta Scientiarum. 2002. 4(3):663-70.

21. Monteiro, LC, Maciel, PP, Bonan, PRF, Ferreira, VYN. Manifestações orais decorrentes da radioterapia em cabeça e pescoço. 60-71. In: Ribeiro, I. A. L., Valença, A. M. G., Bonan, P. R. F. Odontologia na oncologia pediátrica. 2016. João Pessoa, Ideia.

22. Jbam, B, Freire, ARS. Complicações bucais da radioterapia em cabeça e pescoço. Rev Bras Otorinolaringol. 2006. 72(5): 704-8. 
23. Mufeed, A., Jameela, R. V., Isaac, J. K., Vazhagan, V., Shameem, A. Oral complications in an irradiated patient: A case report with review of the literature. SRM J Res Dent Scienc.2015. 6(2): 134-8.

24. Meurman, J. H., Grönroos, L. Oral and dental health care of oral cancer patients: hyposalivation, caries and infections. Oral Oncology. 2010. 46: 464-67.

25. Grimaldi N, Sarmento, V, Provedel, L, Almeida, D, Cunha, S. Conduta do cirurgião dentista na prevenção e tratamento da osteorradionecrose : revisão de literatura. Rev Bras Cancerol.2005.51(4): 319-24.

26. De Castro, RM, Dezotti, M, Azevedo, L, Aquilante, A, Xavier, CR. Atenção odontológica aos pacientes oncológicos antes, durante e depois do tratamento antineoplásico. Rev Odontol UNICID. 2002.14(1):63-74.

27. Schwartz, H, Kagan, R. Osteoradionecrosis of the mandible: scientific basis for clinical staging. Am J Clin Oncol. 2002. 25(2):168-71.

28. Pereira FG, Silva TD, Queiroz FT, Antunes HS, Pontes JR. Leucemia: manifestações orais.

Rev Bras Hematol Hemoter. 2005. 34; Supl. 2: 533-45.

29. Fonseca, M A. Dental care of the pediatric cancer patient. Ped Dent. 2004.26(1): 53-7.

30. Rosales, A.C.M.N. Dental needs in brazilian patients subjected to head and neck radiotherapy. Braz Dent J.2009.20(1): 74-77

Submetido em: 16-2-2017

Aceito em: 14-6-2017 